\title{
Failure to vaccinate and vaccine failure
}

\author{
Access to life-saving vaccines is still a problem for millions around the world, while others endanger public health \\ by refusing available, safe and effective vaccines. Yet some outbreaks increasingly occur in highly vaccinated \\ populations, highlighting the need for further vaccine development to provide long-lasting immunity.
}

Fising ew developments have done more to limit the spread of infectious disease and associated mortality than the advent of vaccination. Since the introduction of mass vaccination, rates of potentially fatal diseases such as polio, measles and diphtheria have plummeted, and smallpox has been successfully eradicated. Vaccines prevent an estimated two to three million deaths each year, and intense efforts are ongoing to develop vaccines against major infectious diseases for which they are not yet available, such as AIDS, malaria and tuberculosis. Multiple factors, including poor healthcare infrastructures and ongoing conflict, limit access to vaccination in some of the hardest hit areas of the world. By contrast, in areas where vaccine access is not an issue, a rise in vaccine hesitancy is a man-made problem that endangers public health, leading to fully preventable outbreaks and deaths. Ironically, the successful reduction in disease incidence achieved through vaccination has likely emboldened the antivaccine movement, as the perception of disease risk and mortality decreased.

Public opinion around vaccine safety is also an issue. For example, misinformation about human papillomavirus (HPV) vaccine safety led to the suspension of a Japanese government campaign promoting mass vaccination that had been launched just months earlier, and to a drop in vaccine coverage (for example, vaccination rates in Sapporo, Japan, went from approximately $70 \%$ to $0.6 \%$ ). These events also fuelled a decline in HPV vaccination rates in other countries, putting an entire generation at an unnecessarily high risk of developing cervical cancer ${ }^{1}$. The recent emergence of measles in industrialized countries is also driven by vaccine hesitancy ${ }^{2}$. Almost 83,000 people contracted measles in Europe in 2018 (the highest number in a decade) and in the United States, the first three months of 2019 have seen more cases than all of 2018 - a stark contrast with the year 2000, when measles was considered to be eliminated from the country. One of six ongoing US outbreaks is proving so hard to curb that local authorities have issued an unprecedented 30-day ban of non-vaccinated children from public spaces. In a move to address this global problem, the WHO has included vaccine hesitancy as one of the ten threats to global health in 2019.
Vaccine misinformation is pervasive and persistent. Despite having been oft proven unfounded, the link between the measles, mumps and rubella (MMR) vaccine and the development of autism still fuels the anti-vaccine movement and continues to be a recurring theme in conversation surrounding vaccines. Multiple studies have disproven this link; for example, a recent study on $>650,000$ children born in Denmark between 1999 and 2010 once again found no increased risk of autism after MMR vaccination, nor triggering of autism in susceptible children ${ }^{3}$. These results follow a study in 2002 of >530,000 Danish children reporting similar conclusions ${ }^{4}$. While additional solid data refuting the autism link is important, the anti-vaccination movement has not waned since the publication of the 2002 study. We, as a society, have the challenge to change the script and strongly advocate the benefits of vaccination while building trust with sceptical communities through engagement rather than indoctrination ${ }^{5}$. The coverage that Hviid et al. ${ }^{3}$ received in the mainstream media and efforts announced over the last few months by companies such as Facebook, Instagram and YouTube to curb the spread of antivaccination content on their platforms are encouraging steps in the right direction.

Scientists are indispensable voices to inform and reassure the public that vaccines are broadly efficacious and safe. They are also essential to address other important gaps beyond those in vaccine administration, such as understanding the causes of vaccine failure. Outbreaks of some diseases, such as whooping cough and mumps, often occur in populations with high vaccine coverage. Specifically, while introduction of the two-dose MMR regime in industrialized countries during the 1980s greatly reduced mumps incidence, the number of outbreaks in vaccinated populations in these regions has steadily increased since the early 2000s (ref. ${ }^{6}$ ). These outbreaks mostly affect young adults who have received a double dose of vaccine, and time since the last dose has been shown to increase the chances of contracting the disease. For example, in some French and Belgian outbreaks the chance of developing mumps increased by
$10-27 \%$ with every year after vaccination ${ }^{6}$, and patients in a US outbreak had more than nine times the risk of mumps if they had received the second MMR dose 13 years or more before the outbreak ${ }^{7}$. Waning immunity (rather than immune escape) is therefore thought to be an important factor behind mumps outbreaks. A third dose of the MMR vaccine was shown to be effective in limiting disease spread in outbreak settings ${ }^{7}$, although antibody levels decrease shortly after the last booster, suggesting that a three-dose vaccination regime would be unlikely to prevent future outbreaks. For whooping cough, where immune escape is likely to also play a role in vaccine failure, waning immunity is still thought to be a crucial factor contributing to outbreaks in vaccinated populations, as the current subunit vaccines seem less potent at generating long-lived immunity than previous whole-cell versions ${ }^{8}$. These findings highlight that more work is needed to better understand the responses to vaccines and develop more immunogenic vaccines that confer longer-lasting immunity.

Vaccines are a success story; that is not in doubt. But this is not a time to rest on past successes and put the advances made so far at risk. We still need new vaccines for infections that are not yet preventable, and continued research into improving existing vaccines to maintain and extend their efficacy. Beyond the bench, increased public health investment should be used to counter misinformation, build public trust in the benefits of vaccination and ensure global access to existing vaccines. It is a challenging road ahead, but one well worth travelling as it will take us all to a healthier world.

Published online: 23 April 2019 https://doi.org/10.1038/s41564-019-0450-5

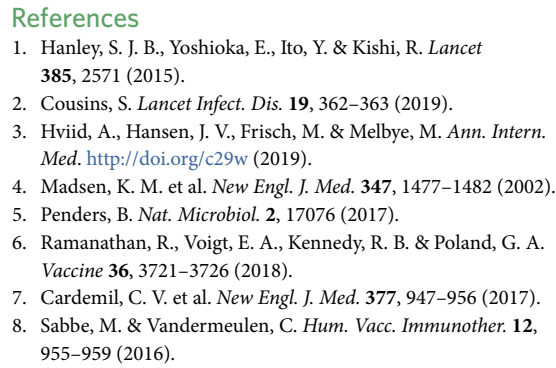

